\title{
A Rederivation of the Alpha Effect in Terms of the Magnetic Fluctuation Spectrum
}

R. K. Keinigs

R. A. Gerwin 
This report was prepared as an account of work sponsored by an agency of the United States Government. Neither the United States Government nor any agency thereof, nor any of their employees, makes any warranty, express or implied, or assumes any legal liability or responsibility for the accuracy, completeness, or usefulness of any information, apparatus, product, or process disclosed, or represents that its use would not infringe privately owned rights. References herein to any specific commercial product, process, or service by trade name, trademark, manufacturer, or otherwise, does not necessarily constitute or imply its endcrsement, recommendation, or favoring by the United States Government or any agency thereof. The views and opinions of authors expressed herein do not necessarily state or reflect those of the United States Governinent or any agency thereof. 
A REDERIVATION OF THE ALPHA EFFECT IN TERYS OF THE

MAGNETIC FLUCTUATION SPECTRUM

by

R. K. Keinigs and R. A. Gerwin

\begin{abstract}
We demonstrate that the alpha effect can be expressed in terms of the integrated current helicity spectrum of the turbulence. This is a much more convenient form than that obtained using a kinematic velocity field description.
\end{abstract}

\title{
I. INTRODUCTION
}

It is a simple exercise to show that a reversed-field pinch (RFP) cannot exist in a cylindrically symmetric, steady state. To demonstrate this let us consider that such a state does exist, and that the magnetic field has the following form:

$$
\underline{\sim}(r)=B_{z}(r) \hat{z}+B_{\theta}(r) \hat{\theta}
$$

Reversal implies that at some radius, $r_{0}, B_{z}(r)$ changes sign; taking $B_{z}(0)>0$, we have

$$
B_{z}\left(r_{0}\right)=0,\left.\frac{\partial B_{z}}{\partial r}\right|_{r_{0}}<0
$$

The condition that the field configuration is steady state implies that the magnetic flux, $\phi(r)$, through a cylindrical cross section of radius, $r$, is constant in time. Therefore $\varepsilon_{\theta}(r)$ must vanish for all $r$ : 


$$
\begin{aligned}
\dot{\phi}(r) & =\int_{A} d \underset{\sim}{d} \cdot \frac{\partial \underset{\sim}{B}}{\partial t}=-\oint d \underset{d}{d} \cdot \underset{\sim}{.} \\
& =-2 \pi r \varepsilon_{\theta}(r)=0 .
\end{aligned}
$$

For anisotropic resistivity the electric field is given by

$$
g(r)=\frac{1}{\sigma_{\perp}} j_{\perp}(r)+\frac{1}{\sigma_{1}} j_{\|}(r)
$$

where $\sigma_{\perp}$ and $\sigma_{\|}$are the perpendicular and parallel conductivities. The current is

$$
j=j_{k} \hat{b}+j_{\perp} \hat{\perp} \text {, }
$$

where

$$
\begin{aligned}
& \hat{b} \equiv \frac{B_{\theta}}{B} \hat{\theta}+\frac{B_{z}}{B} \hat{z}, \\
& \hat{I} \equiv \frac{B_{z}}{B} \hat{\theta}-\frac{B_{\theta}}{B} \hat{z}
\end{aligned}
$$

are unit vectors which lie in the flux surface, $r=$ constant. Employing this representation for $\downarrow$, $\varepsilon_{\theta}$ becomes

$$
\begin{aligned}
\varepsilon_{\theta} & =\frac{1}{\alpha_{\perp}} j_{\perp}^{\theta}+\frac{1}{\sigma_{1}} j_{i}^{\theta} \\
& =\frac{1}{\sigma_{\perp}} \frac{B_{z}}{B}\left(\frac{B_{z}}{B} j_{\theta}-\frac{B_{\theta}}{B} j_{z}\right)+\frac{1}{\sigma_{1}} \frac{B_{\theta}}{B}\left(\frac{B_{z}}{B} j_{z}+\frac{B_{\theta}}{B} j_{\theta}\right) .
\end{aligned}
$$


Since $\varepsilon_{\theta}$ must vanish for all $r$, we may choose $r=r_{0}$; then since $B_{z}\left(r_{0}\right)=0$,

$$
\varepsilon_{\theta}\left(r_{0}\right)=\frac{1}{\sigma_{1}} \frac{B_{\theta}^{2}\left(r_{0}\right)}{B^{2}\left(r_{0}\right)} j_{\theta}\left(r_{0}\right)=\frac{1}{\sigma_{\|}} j_{\theta}\left(r_{0}\right)=0 .
$$

The vanishing of $j_{\theta}\left(r_{0}\right)$ implies $\partial B_{z} /\left.\partial r\right|_{r_{0}}=0$. But this contradicts the initial condition that $r_{0}$ is a reversal point. Therefore this proves that no steady state can exist for a cylindrically symetric RFP. Consequently there must be superimposed on any such steady, symetric field profiles a time-dependent spectrum of fluctuations, or some amount of asymetry. (An example of the latter is the "Helical Ohmic State". 1)

If there does exist in the system a sea of fluctuations it may be possible to suppress the resistive diffusion of the mean magnetic field profiles $\left[B_{z}(r, t), B_{\theta}(r, t)\right]$. It is apparent from the experinental measurements made on ZT-40 and other RFP devices that sone anonaly or "dynamo" is responsible for the relatively long configuration times achieved. Such dynamo action may arise from turbulence. Essentially a dynano is a nechanisa which generates magnetic flux at a sufficiently rapid enough rate to balance or exceed resistive diffusion. In this paper we are prinary interested in the "alpha effect", well-known in dynamo theory. This corresponds to a turbulent electromotive force that is parallel (or antiparallel) to the mean field, $\underline{B}_{0}(x, t)$. The alpha effect is central to the understanding of field generation in astrophysical plasmas and has been proposed as one viable candidate for explaining the sustainment of the reversed field in RFP devices. 2 We demonstrate that $\alpha \equiv \varepsilon_{\text {turb }} \cdot\left(\hat{B}_{0} / B_{0}\right)$ can be conveniently represented in terns of the magnetic fluctuation spectrum. Sone interesting conparisons are made with the results obtained for a via kinenatic dynano theory.

II. THE DYNAMO EQUATION

Our model calculation treats the Idealized geonetry of an unbounded plaswa immersed in a strong magnetic field, $B_{o}(x, t) \hat{z}$. The fluctuating fields are denoted by $\delta \underset{\sim}{(x, t)}, \delta \underline{\sim}(x, t)$, and $1 t$ is assuned that there are no mean flows $\left(V_{0}=0\right)$. Naturally, due to the absence of bounding walls, extrapolation of the results to a real device must be vierred with caution. 
However, we think the physics of even this oversimplified geometry is sufficiently interesting to merit attention. What we want to understand is how turbulence affects the temporal evolution of the cylindrically symmetric, zero-order field profiles. The evolution of the total field, $\underset{\sim}{\mathrm{B}}(\underset{\sim}{x}, t)+$ $\delta \mathrm{B}(\mathrm{x}, t)$, is governed by Faraday's equation. Substituting the electromotive force, $\varepsilon=\eta_{j}-\delta \underset{\sim}{\mathbb{V}} \times \underset{\sim}{\mathrm{B}} / \mathrm{c}$, into this equation yields

$$
\frac{\partial \underline{B}}{\partial t}-\frac{n c^{2}}{4 \pi} \nabla^{2} \underline{B}=\nabla \times(\delta \underset{V}{V} \times \underset{B}{B})
$$

where for simplicity the resistivity, $n$, is assumed uniform. To obtain an equation for the mean field, $\mathrm{\sim}_{0}$, a "two-scale" approximation is made (similar to quasi-linear theory) in which the temporal and spatial scales, $\left(\omega^{-1}, k^{-1}\right)$, associated with the turbulence are considered to be much shorter than the respective scales, $(T, L)$, characterizing $\underset{\sim 0}{B}(x, t) .^{*}$ Angular brackets, \langle\rangle , are used to represent a space-time average over the intermediate scales, $\left(\ell_{0}, \tau\right)$, which satisfy $\left(\omega^{-1}, k^{-1}\right) \ll\left(\ell_{0}, \tau\right) \ll(L, T)$. Applying $\langle>$ to the velocity and magnetic fields implies $\langle\delta \underset{\sim}{\mathbf{V}}\rangle=\langle\delta \underset{\sim}{\mathrm{B}}\rangle=0,\langle\underset{\sim}{\mathrm{B}}\rangle={\underset{\sim}{\mathrm{O}}}_{0}$. In terms of this averaging Eq. ( 1 ) can be separated into two equations, one for the mean and one for the fluctuating magnetic field:

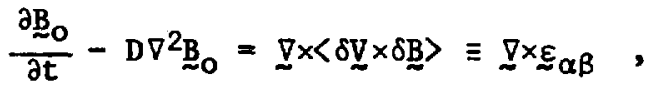

$$
\begin{aligned}
& \frac{\partial \delta \underline{B}}{\partial t}-D \nabla^{2} \delta \underline{B}=\nabla \times\left(\delta \underline{V} \times \underline{B}_{0}\right)+\nabla \times[\delta \underline{V} \times \delta \underline{\underline{P}}-\langle\delta \underline{V} \times \delta \underline{B}\rangle],
\end{aligned}
$$

where

\footnotetext{
*Note that because of the large spatial scale difference separating the turbulent and mean fields this analysis does not apply to global nodes.
} 
$D \equiv \frac{n c^{2}}{4 \pi}$ defines the resistive diffusion coefficient.

Equation (2) determines the evolution of the mean field in the presence of a turbulent emf. Our problem is that of calculating $\varepsilon_{\alpha \beta^{*}}$ (Obviously, if we set $\Sigma_{\alpha \beta}=0$ the magnetic fleld simply resistively decays.) Because ${\underset{\sim}{0}}_{0}(x, t)$ is slowly varying compared with the fluctuating fields it can be considered as being constant and uniform in Eq. (3). In an unbounded system the turbulent emf can be expanded in the form, 3

$$
\varepsilon_{\alpha \beta}^{i}=\alpha_{i j} B_{j}+\beta_{i j k} \frac{\partial}{\partial x_{k}} B_{j}+(\text { higher order terms })
$$

For our straight-field geometry we find

$$
\stackrel{\epsilon}{\alpha \beta}_{\alpha \beta} \alpha \mathrm{B}_{0}+\beta \underset{\sim}{ } \times \underline{B}_{0}+\text { (higher order terms) }
$$

The first term on the RHS of this equation defines the alpha effect; this is an emf directed parallel (or antiparallel) to the field, $\underline{B}_{0}$. The second tern represents the beta effect, a turbulent emf directed along the current. The principal objective of this report is the evaluation of the coefficient, $\alpha$.

Substituting Eq. (4) into Eq. (2)' (1gnoring the higher order terus) yields the dynamo equation,

$$
\frac{\partial \mathrm{B}_{0}}{\partial \mathrm{t}}-\mathscr{\sigma ^ { 2 } \mathrm { B } _ { 0 }}=\alpha \nabla \times \mathrm{B}_{0}
$$

where $\mathcal{O} \equiv D-\beta$. Note that $\beta$ plays the role of a turbulent resistivity. Now assume a solution for $\underset{\sim}{\mathrm{B}}$ of the form, 


$$
{\underset{\sim}{0}}_{0}(\underline{x}, t)=\underline{B}^{1 \underline{K}_{0} \cdot \underline{x}+\gamma t}
$$

where necessar1ly $\left|\underline{\sim}_{0}\right| \ll\left|\underline{k}_{\text {turb }}\right|$ and $\gamma \ll u_{k}$. Then Eq. (5) reduces to

$$
\left(\gamma+B \mathrm{~K}_{0}^{2}\right)_{\mathrm{B}_{0}}=\alpha+\mathrm{J}_{0} \times \mathrm{B}_{\mathrm{O}}
$$

Taking the curl of Eq. (6a) yields

$$
\left(\gamma+\delta \mathrm{K}_{0}^{2}\right) \mathrm{K}_{0} \times \mathrm{B}_{0}=-\alpha+\mathrm{K}_{0}^{2} \mathrm{~B}_{0}
$$

Combining Eqs. (6a) and (6b) results in a quadratic equation for $Y$, the solutions of which are

$$
\gamma_{ \pm}=-D \mathrm{k}_{0}^{2} \pm \alpha \mathrm{k}_{0}
$$

Therefore,

$$
\underline{B}_{0}(\underline{x}, t)=e^{-\delta K_{0}^{2} t}\left(\underline{B}_{+} e^{\alpha k_{0} t}+\underline{B}_{-} e^{-\alpha K_{0} t}\right)
$$

Note that regardless of the'sign of a a growing solution exists provided that

$$
|\alpha|>\mathscr{O}_{\mathrm{K}}
$$

\section{EVALUATING a}

In order to evaluate $\alpha$ a knowledge of the perturbed fielda (or their statistical properties) Is required. The fluctuating magnetic fleld can be deteruined fron Eq. (3), while the velocity field is governed by the womtun equation, 


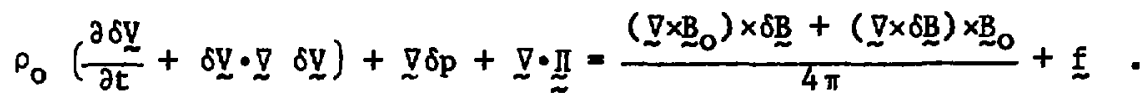

In Eq. (8) $\rho_{0}, \delta p$, and II respectively denote the equilibrium mass density, the perturbed pressure, and the viscous stress tensor. The last term on the RHS, f, represents some unspecified forcing function which drives the turbulence and maintains it at a stationary level. Its presence allows us to assume that, although the system is dissipative, the frequencies of the fluctuating fields can be taken to be real.

A dynamical solution to the dynamo problem requires self-consistent solutions to Eqs. (3) and (8). This necessitates a fully nonlinear treatnent, and one could only hope to tackle such a problem using a 3-D code. If we try to simplify matters by linearizing each of these equations, a dispersion relation results; this yields no information about $\delta V$ or $\delta B$, and hence no information about $\langle\delta \underset{\sim}{\mathbb{V}} \times \delta \underline{\sim}\rangle$. It appears therefore that in order to proceed with evaluating $\varepsilon_{\alpha}$ we must abandon one of these equations. One approach commonly employed is to specify the statistical properties of the velocity field a priori, and in so doing circumvent solving the momentum equation. (This is what is typically referred to as the "kinematic dynamo problem".) Given the velocity field statisitcs Eq. (3) can then be solved for $\delta \underset{\sim}{ }(\underset{\sim}{x}, t)$, and $\langle\delta \underset{\sim}{ } \times \delta B\rangle$ evaluated. What resultis from this approach is an alpha effect expressed in terms of the two-point velocity correlation spectrum. For isotropic turbulence $\S_{\alpha}$ takes the form of a weighted integral of the kinetic belicity (helicity correlated velocity and vorticity, $\langle\delta \underset{\sim}{\nabla} \underset{\sim}{\nabla} \delta \underset{\sim}{\mathrm{V}}\rangle$ ). Unfortunately, this result provides little aid for the experimentalist, since such correlations cannot be measured.

What is needed is a way of expressing $\varepsilon_{\alpha}$ in terus of a more accessible quantity, say, elther the density fluctuation spectrum or the magnetic fluctuation spectrum. We are able to accomplish the lister by using an approach in which the statistical properties of the magnetic turbulence are spectfied. By then solving for $\delta \underline{V}(\underline{x}, t)$ in terms of $\delta B(\underline{x}, t),\langle\delta \underline{V} \times \delta B\rangle$ can be conventently expressed in terms of the integrated current helicity spectrun, $\langle\delta \underset{\sim}{\mathrm{B}} \underset{\sim}{\nabla} \times \delta \mathrm{B}\rangle$. Although this approach is also kinematic, 1.e., the actual dynanics of the turbulence is 1gnored, we reserve the nomenclature, "kineatic dynano," for the more standard velocity-fleld approach. 
We begin by considering Eq. (3), dropping the second term on the RHS. This approximation is known as "first-ordex smoothing"; 4 by ordering the various terms in Eq. (3) one finds that neglecting this term requires that the phase velocity of the perturbation be much larger than the field velocity, i.e., $|\omega / \mathrm{k}| \gg|\delta \underline{V}|$. The reduced form of $\mathrm{Eg}$. (3) then becomes

$$
\frac{\partial \delta \underset{\sim}{\mathrm{B}}}{\partial t}-\mathrm{D} \nabla^{2} \delta \underset{\sim}{\mathrm{B}}=-{\underset{\sim}{\mathrm{B}}}_{\mathrm{O}}(\underset{\sim}{\nabla} \cdot \delta \underset{\sim}{\mathrm{V}})+\left({\underset{\sim}{\mathrm{O}}}_{\mathrm{O}} \cdot \nabla\right) \delta \underset{\sim}{\mathrm{V}}
$$

( B $_{0}$ is considered uniform.) Fourler transforming Eq. (9) yields

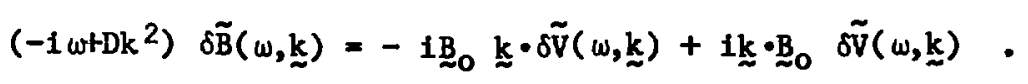

At this stage what is usually done in kinematic dynamo theory is to assume incompressibility and solve Eq. (10) for $\delta \tilde{B}(\omega, k)$. Instead of following this procedure we rewrite Eq. (10) in the form,

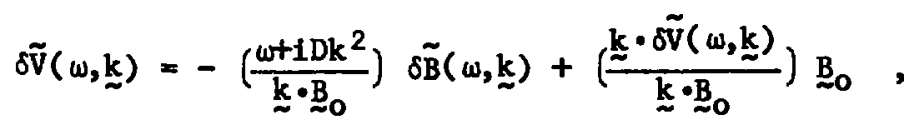

and solve Eq. (11) for $\delta \tilde{V}(\omega, \underline{k})$. If here we impose $k \cdot \delta \tilde{V}=0$ a simple solution for $\delta \tilde{V}(\omega, \underline{k})$ is obtained. However, this is unnecessary for evaluating $\alpha$ : recall that $\alpha$ is proportional to the turbulent emf that is directed along ${ }_{\sim 0}$. Since the second term in Eq. (11) is parallel to B $_{0}$ this contribution to oV cannot contribute to $\alpha$. Neither will it contribute to a perpendicular emf, since by symmetry this must vanish. (Note that in a bounded systen symetry cannot be invoked to guarantee $\varepsilon_{\perp}=0$, and this second term may becone important. For a discussion of the "cross-field" alpha effect see Los Alanos report LA-9290-MS.5) Given that only the perpendicular conponent of $\delta \tilde{F}$ contributes to $\S_{\alpha}$ we may, without 1088 of generality, rewrite Eq. (11) as 


$$
\delta \tilde{V}(\omega, \underline{k})=-\left(\frac{\omega+i D k^{2}}{k_{z_{0}} B_{0}}\right) \delta \tilde{B}_{\perp}(\omega, k)+\delta V_{\nu}(\omega, k) \hat{B}_{0} .
$$

Now that $\delta \tilde{V}(\omega, k)$ has been determined, the electromotive force, $\varepsilon_{z}$, can be evaluated:

$$
\begin{aligned}
\varepsilon_{z}= & \operatorname{Re} \varepsilon_{z j \ell}\left\langle\delta V_{j}(\underline{x}, t) \delta B_{\ell}(\underline{x}, t)\right\rangle \\
= & -\operatorname{Re} \varepsilon_{z j \ell} \frac{1}{B_{0}} \int_{-\infty}^{\infty} d \omega d \omega^{\prime} \int d^{3} k d^{3} k^{\prime} \exp \left[i\left(\underline{k}-\underline{x}^{\prime}\right) \cdot x\right. \\
& \left.-i\left(\omega-\omega^{\prime}\right) t\right] \cdot \frac{\omega+i D k^{2}}{k_{z}}\left\langle\delta B_{j}(\omega, \underline{k}) \delta B_{\ell}^{*}\left(\omega^{\prime}, \underline{k}^{\prime}\right)\right\rangle .
\end{aligned}
$$

This can be expressed in terms of the two-point spectral tensor for the magnetic correlations by rewriting the ensemble average in Eq. (13) as

$$
\begin{aligned}
& \left\langle\delta B_{j}(\omega, \underline{w}) \delta B_{\ell}^{*}\left(\omega^{\prime}, \underline{w}^{\prime}\right)\right\rangle=\int d^{3} x d^{3} x^{\prime} \int_{-\infty}^{\infty} d t d t^{\prime} \exp \left[i\left(\omega t-\omega^{\prime} t^{\prime}\right)\right. \\
& \left.-1\left(\underset{\sim}{k} \cdot \underline{x}^{x}-\underline{w}^{\prime} \cdot \underline{x}^{\prime}\right)\right] \cdot\left\langle\delta B_{j}(\underline{x}, t) \delta B_{\ell}^{*}\left(\underline{x}^{\prime}, t^{\prime}\right)\right\rangle \text {. }
\end{aligned}
$$

Assuming the turbulence to be homogeneous and stationary, and making the change of variables, $\left(\underline{\sim}=x^{-} \underline{x}^{\prime}, \because=t-t^{\prime}\right)$, reduces this to the desired form,

$$
\begin{aligned}
& \left\langle\delta B_{j}(\omega, k) \delta B_{\ell}^{*}\left(\omega^{\prime}, k^{\prime}\right)\right\rangle=\int_{-\infty}^{\infty} d^{3} r d \tau \int_{-\infty}^{\infty} d^{3} x d t \exp \left[i\left(\omega-\omega^{\prime}\right) t\right. \\
& \left.-i\left(\underline{\underline{k}}-\underline{k}^{\prime}\right) \cdot \underline{x}\right] \cdot \exp \left[-i\left(k^{\prime} \cdot \underline{\underline{r}}-\omega^{\prime} \tau\right)\right] . \\
& \left\langle\delta B_{j}(x, t) \delta B_{\ell}^{*}(x+\underline{x}, t+\tau)\right\rangle \text {. } \\
& \equiv \phi_{j \ell}(\omega, \underline{k}) \delta\left(\omega-\omega^{\prime}\right) \delta\left(\underline{k}-\underline{k}^{\prime}\right) .
\end{aligned}
$$

The function, $\phi_{j l}(\omega, \underline{k})$, defines the Fourier spectral tensor for the two-point magnetic correlations. It is formally defined as 6 


$$
\phi_{j \ell}(\omega, \underline{\xi})=\int d^{3} r d t \exp [i(\omega t-\underline{w} \cdot \underline{r})] \cdot\left\langle\delta B_{j}(x, t) \delta B_{\ell}^{*}(\underline{x}+\underline{r}, t+\tau)\right\rangle
$$

The fact that the turbulence is homogeneous and stationary means that the cern in $\langle>$ depends only upon $r$ and $\tau$. If a bounding wall is introduced the condition of homogeneity no longer applies. The spatial correlations of magnetic field fluctuations in bounded systems has been investigated by Turner. ${ }^{7}$ Substituting Eq. (14) into Eq. (13) yields for the coefficient, $a$,

$$
\alpha=-\frac{\operatorname{Re}}{B_{0}^{2}} \varepsilon_{z j \ell} \int_{-\infty}^{\infty} d \omega \int d^{3} k \frac{\omega+1 D k^{2}}{k_{z}} \phi_{j \ell}(\omega, k)
$$

The statistical properties of the magnetic turbulence are manifest in the correlation tensor, $\phi_{j \ell}(\omega, k)$. This tensor must satisfy two conditions:

$$
\begin{array}{ll}
\text { (i) reality } \quad \Rightarrow \quad \phi_{j \ell}=\phi_{\ell j}^{*}, \text { and } \\
\text { (1i) solenoidal } \Rightarrow \quad k_{j} \phi_{j l}=0 .
\end{array}
$$

This second condition arises from the requirement $\underset{\nabla}{\bullet} \delta \underline{\sim}=0$. If $\phi_{j l}$ represented velocity correlatiors ther condition (i1) would only apply if $\underline{\nabla} \cdot \delta \mathrm{V}=0$. The most gelieral form for $\phi_{j \ell}$ that satisfies (i) and (ii) is given by

$$
\begin{aligned}
\phi_{j \ell}(\omega, k) & =\frac{G(\omega, k, \mu)}{4 \pi k^{2}}\left(\delta_{j \ell}-\frac{k_{j} k_{\ell}}{k^{2}}\right) \\
& +1 \frac{H(\omega, k, \mu)}{8 \pi k^{4}} \varepsilon_{j n \ell} k_{n},
\end{aligned}
$$

where $\mu \equiv \hat{k} \cdot \hat{z}$. The $\mu$ dependence of the functions, $G$ and $H$, has been introduced in order to admit the possibility of the mean field inposing a 
preferred axis of symmetry on the turbulence. If the turbulence is truly isotropic (spherically symmetric) then $G$ and $H$ are $\mu$ independent. $A$ reasonable parameter for distinguishing these two cases is $\Omega \tau_{1}$, the ratio of the ion-ion collision time $\left(\tau_{1}\right)$ to the ion gyroperiod $\left(\Omega^{-1}\right)$. If $\Omega \tau_{1} \ll 1$ then ${\underset{\sim}{0}}_{0}$ is considered "weak" and has negligible influerice on the turbulence. In the opposite 1imit, $\Omega \tau_{1} \gg 1$, the mean field is considered "strong" and may - ve a considerable effect on the dynamics. One obvious effect is a rhange in is form of the viscous stress tensor. For this latter case the $\mu$ dependence must be retalned.

The magnetic energy and current helicity spectral densities are $r \geq$ lated to the functions, $G$ and $H$, via

$$
\begin{aligned}
& E(\omega, k)=\frac{1}{2} \int d \underset{\sim}{S} \phi_{i 1}(\omega, k)=\frac{1}{2} \int_{-1}^{1} d \mu G(\omega, k, \mu), \\
& F(\omega, k)=1 \int d \underset{\sim}{S} \varepsilon_{1 n \ell} k_{n} \phi_{i \ell}(\omega, k)=\frac{1}{2} \int_{-1}^{1} d \mu H(\omega, k, \mu),
\end{aligned}
$$

where dS denotes an integration over a spherical surface of radius, $k$. It is in terms of these quantities, $E$ and $F$, that we wish to express $\alpha$. In the case of fully isotropic turbulence $G$ and $H$ are identically equal to the energy and helicity spectral densities. However, since we are interested in strong wean fields we retain the $\mu$ dependence.

If we now substitute Eq. (16) into Eq. (15) a very simple form for $\alpha$ emerges. [Note that due to the permutation synbol, $\varepsilon_{2 j l}, G(\omega, k, \mu)$ will not contribute.]

$$
\begin{gathered}
\alpha=-\frac{R e}{B_{0}^{2}} \varepsilon_{z j \ell} \int_{-\infty}^{\infty} d \omega \cdot 2 \pi \int_{0}^{\infty} d k k^{2} \int_{-1}^{1} d \mu \frac{\omega+1 D k^{2}}{k_{z}} . \\
i \varepsilon_{j n l} k_{n} \frac{H(\omega, k, \mu)}{8 \pi k^{4}}
\end{gathered}
$$




$$
\begin{aligned}
& =\frac{D}{B_{0}^{2}} \int_{-\infty}^{\infty} d \omega \int_{0}^{\infty} d k \frac{1}{4} \int_{-1}^{1} d \mu \varepsilon_{z j l} \varepsilon_{j n \ell} \frac{k_{n}}{k_{z}} H(\omega, k, \mu) \\
& =\frac{-D}{B_{0}^{2}} \int_{-\infty}^{\infty} d \omega \int_{0}^{\infty} d k \frac{1}{2} \int_{-1}^{1} d \mu H(\omega, k, \mu), \text { or } \\
\alpha & =\frac{-D}{B_{0}^{2}} \int_{-\infty}^{\infty} d \omega \int_{0}^{\infty} d k F(\omega, k) .
\end{aligned}
$$

Equation (18) is our principal result. It indicates that two conditions are necessary in order for fine-scale turbulence to result in an alpha effect; these are

(i) The system must be dissipative. Here the dissipation enters through the resistivity (D). The resistivity provides a necessary phase shift between $\delta \underline{\mathbb{V}}$ and $\delta \underline{\mathrm{B}}$ without which $\underline{\varepsilon}_{\text {turb }} \equiv 0$. (This is a we11-known result of kinematic dynamo theory also.)

(11) A finite current helicity, $F(\omega, k) \neq 0$, is required for dynamo action. This is equivalent to the statement that the magnetic turbulence lacks reflexional symetry. A comment on compressibility: observe that in obtaining Eq. (18) we have not required $\underset{\nabla}{\nabla} \delta \mathbf{V}=0$. However, since compressibility does explicitly enter this equation its effect, if any, must be buried in the helicity spectrum.

IV. COMPARISON WITH KINEMATIC DYNAMO THEORY

At this point it is appropriate to compare Eq. (18) and 1 ts inplications for dynamo action with the results that are obtained for the alpha effect employing the more standard kinematic approach. For a detalled expose on kinematic dynamo theory the reader is referred to the book by Moffatt ${ }^{4}$ from which the following results are extracted.

In contrast to our approach kinenatic dynano theory assunes that the statistical properties of the velocity field are known. Faraday's equation is then solved for $\delta \underline{B}(\omega, \underline{k})$, after which $\langle\delta \underline{\delta} \times \delta \mathrm{B}\rangle$ can be evaluated. The analjais is similar to ours, except inconpreselbility is assuned. (If $\nabla \cdot \delta \underline{0} \neq 0, \phi_{j l}$ 
does not satisfy the solenoidal condition, and its form becomes mathematically more complicated.)

Given $\underset{\nabla}{\sigma} \delta \mathrm{V}=0$ and that the velocity fluctuations are isotropic $\left(\Omega \tau_{1} \ll 1\right), \alpha$ has the following fora:

$$
\alpha=-\frac{D}{3} \int_{-\infty}^{\infty} d \omega \int_{0}^{\infty} d k \frac{k^{2}}{\omega^{2}+D^{2} k^{4}} \bar{F}(\omega, k),
$$

where $\vec{F}(\omega, k)$ defines the kinetic helicity spectral density. Once again, in order to have dynamo action dissipation is zequired, as is helicity (this time in the form of a finite correlation between the velocity and vorticity fluctuations). Equation (19) is complicated however by the weighting factor present in the integrand.

If we next consider the strong field limit $\left(\Omega \tau_{i} \gg 1\right)$ it is straightforward to show that $\alpha$ now assunes the form,

$$
\alpha=-\frac{D}{3} \int_{-\infty}^{\infty} \mathrm{d} \omega \int_{0}^{\infty} \mathrm{dk} \frac{\mathrm{k}^{2}}{\omega^{2}+\mathrm{D}^{2} \mathrm{k}^{4}} \cdot \frac{1}{2} \int_{-1}^{1} \mathrm{~d} \mu \mu^{2} \overline{\mathrm{H}}(\omega, k, \mu) .
$$

Provided $\overline{\mathrm{H}}$ is $\mu$ Independent this reduces to Eq. (19); but for the case of a strong magnetic field this is not likely, and the previous conclusion that $\alpha$ requires kinetic helicity may not apply. This is for the following reason: when there exists a preferred axis of symmetry the kinetic helicity spectrum is given by [see Eq. (19b)]

$$
\bar{F}(\omega, k)=\frac{1}{2} \int_{-1}^{1} d \mu \overline{\mathrm{H}}(\omega, k, \mu)
$$

Comparing this to the $\mu$ integral in Eq. (20) it appears possible that $\alpha$ ay be finite, while at the same time the helicity, $\bar{F}(\omega, k)$, vanishes. This is due to the weighting of the $\mu$ integral in $\mathrm{Eq}$. (20). However, according to Eq. (18), regardless of the symmetry, a net current helicity is required for finite a. This observation raises an interesting question: "In an electrically 
conducting fluid are the current and kinetic helicities necessarily proportional?" If not, one must conclude that it is the cutrent helicity, and not the kinetic helicity, which is the necessary physical quantity underlying dynamo action.

It should be pointed out that for the geometry we have considered it is difficult to envision how helicity can be generated. It may be possible to preferentially excite one sign of helicity if a small amount of shear is introduced into ${\underset{\sim}{\mathrm{B}}}_{0}(x, t)$. This could have very interesting implications for RFP and is currently being investigated.

V. SCALING OF $\left|\delta \underset{\sim}{\mathrm{B}} / \mathrm{B}_{0}\right|$ WITH MAGNETIC REYNOLD'S NUMBER

Now that a form for $\alpha$ has been optained [Eq. (18)] it can be used to estimate the level of magnetic fluctuations required to compensate for resistive losses. According to Eq. (7) resistive diffusion can be balarced iy dynamo action provided that $|\alpha|>\mathscr{V}_{\mathrm{K}}$. For simplicity we will ignore $B$, so $\vartheta \approx$ D. Then Eq. (18) implies that for steady state we must have

$$
\int_{-\infty}^{\infty} d \omega \int_{0}^{\infty} d k \frac{F(\omega, k)}{B_{0}^{2}}=k_{0}
$$

where $K_{0}^{-1} \equiv a$ is a characteristlc scale length for the mean field, Do $_{0}$ Since the functional form of $F(\omega, k)$ is unknown (a consequence of not having solved the full dynamical problen). we make a guess: assume that the turbulence is peaked in a narrow band about the frequency and wave vector pair $\left(\omega_{0}, k_{0}\right)$. Then the LHS of Eq. (21) can be approximated by $\mathcal{f}\left(\omega_{0}, k_{0}\right) / \mathrm{B}_{0}^{2}$, where $\mathcal{J}\left(\omega_{0} k_{0}\right)$ now represents the current helicity in "real" space. $f\left(\omega_{0}, k_{0}\right)$ can be expressed as some fraction, $\Delta$, of the maximal helicity, where $F_{\text {max }}$ is simply equal to $k_{0} E\left(\omega_{0}, k_{0}\right)$, $E$ being the magnetic fluctuation energy. Using these arguments,

$$
f\left(\omega_{0}, k_{0}\right)=\Delta k_{0} E\left(\omega_{0}, k_{0}\right)=\Delta k_{0} \frac{\left|\delta B\left(\omega_{0}, k_{0}\right)\right|^{2}}{8 \pi},
$$


and the condition for steady state becomes

$$
\frac{\Delta}{8 \pi} k_{0} \frac{\left|\delta B\left(\omega_{0}, k_{0}\right)\right|^{2}}{B_{0}^{2}}=k_{0}=\frac{1}{a}
$$

The required fluctuation level is therefore

$$
\left|\frac{\delta B_{1}}{B_{0}}\right|=\left(\frac{8 \pi}{\Delta}\right)^{1 / 2}\left(\frac{1}{k_{0} a}\right)^{1 / 2} .
$$

The problem reduces to that of finding an appropriate wave vector for the turbulence. As an example consider a spectrum of Alfvén waves centered about

$$
\frac{\omega_{0}}{k_{0}}=C_{A}
$$

For very short wavelengths the resistive damping decrement increases until it is of the same magnitude as the real frequency, $\omega_{0}$. We may then conjecture that $\omega_{0} \sim D_{k_{0}}^{2}$. Using this approximation Eq. (23) yields for $k_{0}$,

$$
k_{0}=\frac{C_{A}}{D}
$$

Substituting this into Eq. (22) gives

$$
\left|\frac{\delta B}{B_{0}}\right|=\left(\frac{8 \pi}{\Delta}\right)^{1 / 2}\left(\frac{D}{a^{2}} \frac{a}{C_{A}}\right)^{1 / 2}=\left(\frac{8 \pi}{\Delta}\right)^{1 / 2} s^{-1 / 2},
$$

where $S=\tau_{R} / \tau_{A}$ defines the nagnetic Reynold's number. This inverse scaling 
of fluctuation level with $S$ becomes more favorable as the plasma temperature increases. Bunting et al. ${ }^{8}$ have obtained a similar s-scaling for the case of growing resistive kink modes. The scaling obtained in Eq. (24) may differ if the frequency, $\omega_{0}$, is determined by something other than the resistive cut-off f requency.

VI. CONCLUSION

We have shown that the alpha effect in a simple straight-field geometry Is directly related to the integrated current helicity spectrum. The form of $\alpha$ is independent of the symetry imposed on the turbulence by the magnetic field. We have estimated the magnetic fluctuation level required to give efficient dynamo action and found that it scales with magnetic Reynold's number as $\left|\delta \mathrm{B} / \mathrm{B}_{\mathrm{O}}\right| \sim \mathrm{s}^{-1 / 2}$.

APPENDIX A

AMPLIFICATION OF FORCE-FREE FIELDS

It so happens that for force-free fields amplification requires that $a$ and the current helicity of the mean field have the sane sign. To show this consider a force-free field satisfying

$$
\underset{\mathbb{N}_{0}}{\nabla \times \mathrm{B}_{0}}=\mathrm{KB}_{0}
$$

The dynamo equation [Eq. (5)] can then be expressed as

$$
\stackrel{B}{B}_{0}=\frac{\alpha-K \mathscr{E}}{\gamma} \underset{\nabla}{ } \times \underline{B}_{0},
$$

where again it is assumed that $\mathbb{\sim}_{0}$ has the temporal dependence, $\exp (\gamma t)$. How $\operatorname{dot}$ Eq. (A2) with Bo: $_{0}$ 


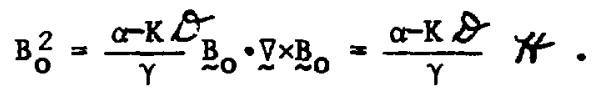

If defines the current helicity associated with the mean magnetic field. It was shown in Sec. II that in order to amplify $\mathrm{B}_{0}(\gamma>0)|\alpha|$ must be greater than $D_{\mathrm{K}}$, and $\underset{\sim 0}{\mathrm{~B}}$ must have a preferred helicity. Let us assume that $|\alpha|>\delta \mathrm{K}$, and consider the two possibilities for $A$ : (1) $\Psi>0$ and (2) $\not<0$. For $\not>0$, since the LHS of Eq. (A3) is positive, we must have $(\alpha-K O) / \gamma>0$. If $\alpha<0$, then $\gamma<0$ and ${\underset{\sim}{0}}_{0}$ decays with time. If $\alpha>0$, then $\gamma>0$ and $\underset{\sim}{\mathrm{B}}$ is amplified. Case (2) can be treated in exactly the same manner with analogous results: $\alpha>k \theta \Rightarrow \underline{B}_{0}$ decays, $\alpha\left\langle-k \sigma \Rightarrow B_{0}\right.$ grows. Hence amplification of the mean fleld requires that $\alpha$ and $t<$ have the same sign. '(This observation was pointed out to the authors by Leaf Turner.) What is interesting about this result is that it indicates that amplification of a force-free field requires that the turbulent current heliclty have the opposite sign of the mean-field heliclty [see Eq. (18)]. We have not been able to show that this also holds for amplification of nonforce-free fields.

\section{REFERENCES}

1. R. Y. Dagazian, in "Proceedings of the Reversed Field PInch Theory Workshop," Los Alamos National Laboratory report LA-8944-C (1982).

2. C. G. Gimblett and M. L. Watkins, in Controlled Fusion and Plasna Physics, Proc. 7th European Conf.: Lausanne, 1975, V. 1, p. 103.

3. F. Krause, K. -H. Rädler, and M. Steenbeck, "The Turbulent Dynano," NCAR Technical Notes NCAR-TN/IA-60 (1971).

4. H. K. Moffatt, Magnetic Field Generation in Electrically Conducting Fluids (Cambridge Univ. Press, 1978).

5. R. A. Gerwin and R. K. Kelnigs, "Dynamo Theory: Can Amplification of Magnetic Field Profiles Arise from a Cross-Fleld Alpha Effect?," Los Alamos National Laboratory report LA-9290-MS (April 1982).

6. G. K. Batchelor, Homogeneous Turbulence (Cambridge UnIv. Press, 1953).

7. Leaf Turner, "Statistical Mechanics of a Bounded Magnetofluid," (to be published).

8. C. A. Bunting, C. W. Cowers, K. Ogawa, D. C. Robinson, and D. C. Watts, in "Controlled Fusion and Plasma Physics," Proc. 8th European Conf., Prague, 1977 , v. 1, p. 79 . 


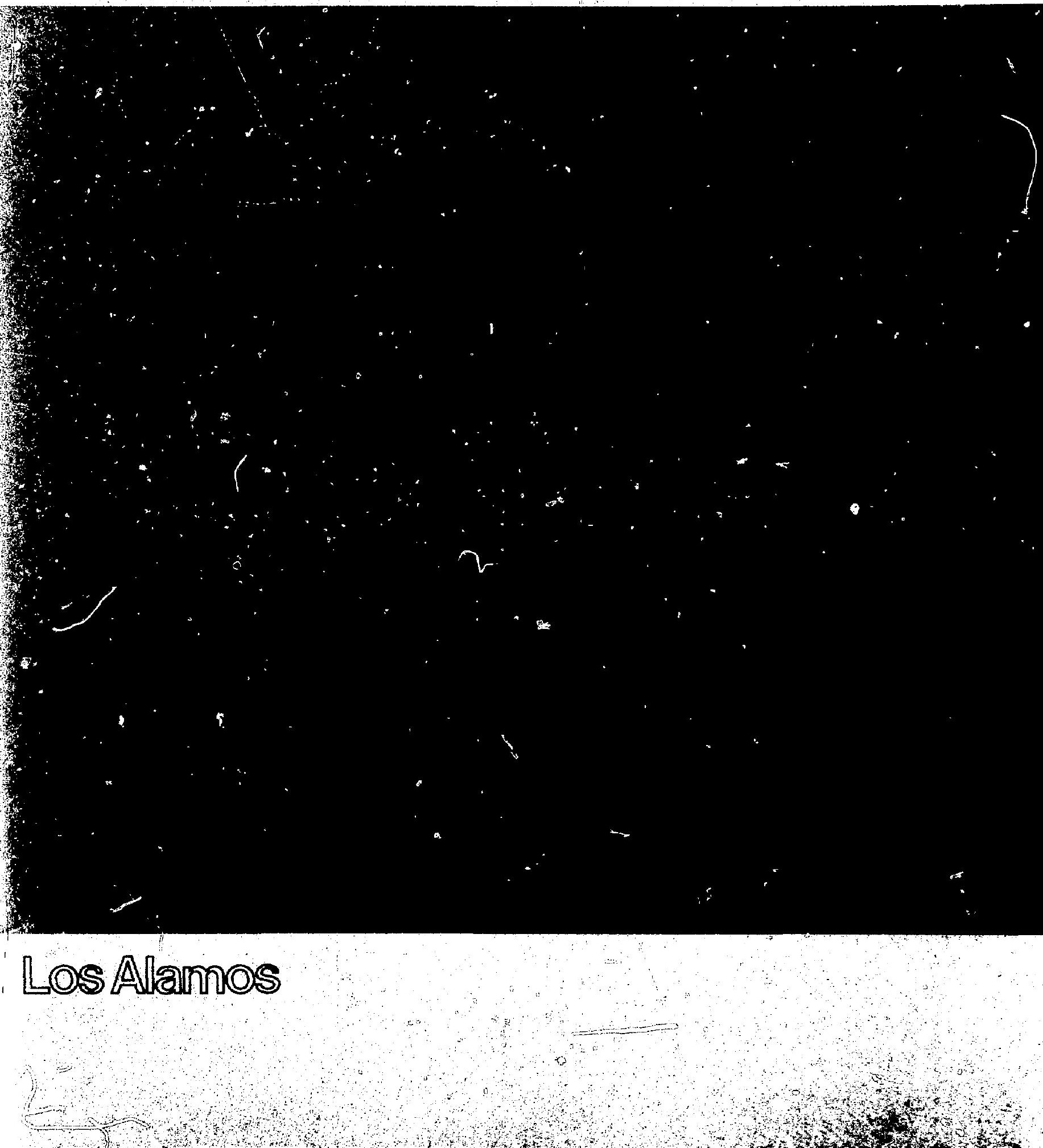

